\title{
The Stephani Universe, K-Essence and Strings in the 5th Dimension
}

\author{
Gary Bruce Tupper ${ }^{1 *}$, Mark Marais ${ }^{2}$, Jose Abdella Helayël-Neto ${ }^{3}$ \\ ${ }^{1}$ Department of Physics, University of Cape Town, Private Bag, Rondebosch, South Africa \\ ${ }^{2}$ Cape Peninsula University of Technology, Symphony Way, Bellville, South Africa \\ ${ }^{3}$ Centre for Brazilian Research in Physics, Rua Dr. Xavier Sigaud, Rio de Janeiro, RJ, Brazil \\ Email: gary.b.tupper@gmail.com, maraism@cput.ac.za, helayel@cbpf.br
}

How to cite this paper: Tupper, G.B., Marais, M. and Helayël-Neto, J.A. (2021) The Stephani Universe, K-Essence and Strings in the 5th Dimension. Open Access Library Journal, 8: e3654.

https://doi.org/10.4236/oalib.1103654

Received: July 20, 2021

Accepted: August 17, 2021

Published: August 20, 2021

Copyright (C) 2021 by author(s) and Open Access Library Inc.

This work is licensed under the Creative Commons Attribution International License (CC BY 4.0).

http://creativecommons.org/licenses/by/4.0/

\begin{abstract}
The Stephani universe is an inhomogeneous alternative to $\Lambda$ CDM. We show that the exotic fluid driving the Stephani exact solution of Einstein's equations is an unusual form of k-essence that is linear in "velocity". Much as the Stephani universe can be embedded into (a section of) flat 5-d Minkowski space-time, we show that the k-essence obtains through dimensional reduction of a 5-d strongly coupled non-linear "electrodynamics" that, in the empty Stephani universe, corresponds to space filling magnetic branes in string/M-theory.
\end{abstract}

\section{Subject Areas}

Modern Physics

Keywords

Inhomogeneous Cosmology, K-Essence

\section{Introduction}

The discovery [1] [2] that distant supernovae are dimmer than they would be in an Einstein-de Sitter universe which has forced a fresh contemplation of issues almost as old as general relativity itself. If accelerated Hubble expansion driven by a cosmological constant, $\Lambda$ is the explanation, one is left the difficult task of explaining why $\Lambda$ is infinitesimally small in natural units of the Planck mass, and just such as to reveal itself in the present epoch [3].

A much discussed alternative is to assume that the cosmological constant vanishes and the acceleration is due to the nonlinearity of the Einstein equations: since the present universe is only homogeneous and isotropic on average, aver-

*Associate member National Institute for Theoretical Physics. 
aging of the Einstein equations will yield the usual FRW model equations plus corrections from "back-reaction" [4]. It is easy to realise this by constructing spherical LTB metrics that can account for the supernova data [5], however, such models assume a co-moving co-ordinate system and rely upon significant shear. Recalling that the inhomogeneity in the solar system is far larger than the cosmological one, yet is readily treated by post-Newtonian approximation, suggests that corrections to the usual linearly perturbed FRW model will be similarly small, and indeed this proves to be the case [6].

Still, the large-scale homogeneity of the universe is much less observationally secure than its isotropy [7], so suggesting the simplest inhomogeneous but isotropic and shear-free generalization of the FRW metric [7]:

$$
\mathrm{d} s^{2}=N(t, r)^{2} \mathrm{~d} t^{2}-R(t, r)^{2} \mathrm{~d} \vec{x}^{2}
$$

Assuming a perfect fluid source, $T_{\mu v}=(\rho+p) u_{\mu} u_{v}-g_{\mu v} p$, the resulting Einstein equations ${ }^{1}$ were first solved by Wyman [8]; the vanishing of the off-diagonal components of the source in co-moving co-ordinates provides:

$$
\frac{R_{, t}}{N R}=\frac{\dot{R}}{R}=H(t), N=\frac{R_{, t}}{H(t) R}
$$

Herein, e.g. $\dot{R}=N^{-1} R_{, t}$ indicates the proper time derivative, and $H(t)$ is a function of integration. Further, as $T_{i}^{j}=-p \delta_{i}^{j}$ implies $G_{r}^{r}=G_{\theta}^{\theta}=G_{\phi}^{\phi}$, one is led to the "pressure isotropy equation":

$$
R^{\prime \prime}-2 \frac{R^{\prime 2}}{R}-\frac{R^{\prime}}{r}=\frac{1}{2} f(r)
$$

The "primes" here denote partial derivatives with respect to $r$ and $f(r)$ is another integration function. The remaining Einstein equations can be expressed in the Friedman-like form:

$$
\begin{aligned}
& 3 H^{2}=\rho+\frac{1}{R^{2}}\left[\frac{2 R^{\prime \prime}}{R}-\left(\frac{R^{\prime}}{R}\right)^{2}+\frac{4}{r} \frac{R^{\prime}}{R}\right] \\
& \dot{\rho}+3 \frac{\dot{R}}{R}[\rho+p]=0
\end{aligned}
$$

Wyman's objective was to obtain solutions for a barotropic equation of state $p=p(\rho)$, so that he excluded a solution that would later be rediscovered by Stephani [9]

$$
f=0, \quad H=\frac{a_{, t}}{a}, \quad R=\frac{a(t)}{1+k a(t) r^{2} / 4}=a N
$$

The corresponding energy density and pressure follow as:

$$
\rho=3\left[H^{2}+\frac{k}{a^{2}}\right], \quad p=-\rho-\frac{1}{3} \frac{1+k a(t) r^{2} / 4}{H} \rho_{, t}
$$

That is to say, the energy density is homogeneous while the pressure is inhomogeneous. Thus, while the Stephani model has been considered as an alterna-

${ }^{1}$ We use units $8 \pi G=c=1$. 
tive to the $\Lambda C D M$ model [10], its viability is obscured by the question: what is the nature of the perfect fluid source having these unusual properties?

In this paper we will provide an answer to the aforementioned question: the source is a particular case of "k-essence" [11], having a Lagrangian density that is linear in the "velocity". Moreover, just as the Stephani metric is exceptional in that it can be embedded into 5-dimensional Minkowski space [9], so too can the k-essence source be lifted to a 5-dimensional nonlinear "electrodynamics".

The remainder of this paper is organised as follows: in Section 2 we briefly review and reformulate k-essence in a way that makes the choice of Lagrangian density yielding (6) self-evident. Then in Section 3 we show how general $\mathrm{k}$-essence models can be obtained by dimensional reduction from 5-dimensional nonlinear electrodynamics. Finally, our conclusions are presented in Section 4.

\section{K-Essence and the Stephani Universe}

K-essence [11] is simply the most general model Lagrangian density for a scalar field $\varphi$ involving its first covariant derivative $\varphi_{, \mu}$. We take the derivative to be time-like so

$$
\mathcal{L}=\mathcal{L}\left(\varphi, Y \equiv \sqrt{g^{\mu v} \varphi_{, \mu} \varphi_{, v}}\right)
$$

The use of the "velocity" $Y$ instead of the usual $X=g^{\mu v} \varphi_{, \mu} \varphi_{, v}$ as the kinematic variable considerably simplifies and clarifies the subsequent treatment, e.g. the stress-energy tensor takes the perfect fluid form $T_{\mu v}=(\rho+p) u_{\mu} u_{v}-g_{\mu \nu} p$ with the identifications

$$
u_{\mu}=\varphi_{, \mu} / Y, \quad p=\mathcal{L}, \quad \rho=Y \mathcal{L}_{, Y}-\mathcal{L}
$$

Indeed, in co-moving coordinates $Y=\dot{\varphi}$ and $u_{\mu}=\delta_{\mu}^{0} / N$, while the energy density is evidently just the Hamiltonian. The $\varphi$ field equation here reads

$$
\left(\mathcal{L}_{, Y} g^{\mu v} \varphi_{, v} / Y\right)_{: \mu}=\mathcal{L}_{, \varphi}
$$

Imposing the nominal requirements of stability and causality, the adiabatic speed of sound squared is given $b^{2}$

$$
0 \leq c_{s}^{2}=\frac{p_{, Y}}{\rho_{, Y}}=\frac{\mathcal{L}_{, Y}}{Y \mathcal{L}_{, Y Y}} \leq 1
$$

That is to say, the Lagrangian density must satisfy the inequalities: $\mathcal{L}_{, Y} \geq 0 \quad \&$ $\mathcal{L}_{, Y Y} \geq 0$.

Particular classes of $\mathrm{k}$-essence are factorizable models, $\mathcal{L}(\varphi, Y)=-V(\varphi) F(Y)$ (which includes tachyon models [12] [13] for $F(Y)=\sqrt{1-Y^{2}}$ and the Chaplygin gas [14] in the subcase of constant potential), and purely kinetic models (such as Scherrer's [15] model, $\left.\mathcal{L}(Y) \simeq \mathcal{L}\left(Y_{0}\right)+\mathcal{L}^{\prime \prime}\left(Y_{0}\right)\left(Y-Y_{0}\right)^{2} / 2\right)$. Herein we are interested in models of the type [16]:

$$
\mathcal{L}(\varphi, Y)=F(Y)-V(\varphi)=p \Rightarrow \rho=Y F^{\prime}(Y)-F(Y)+V(\varphi)
$$

${ }^{2}$ For a derivation and further discussion see [12]. 
This is because in co-moving co-ordinates $\varphi$ is a function of $x^{0}=t$ only, so that the energy density will be homogeneous if $Y F^{\prime}(Y)-F(Y)=0$, i.e. for some constant $K$

$$
\mathcal{L}(\varphi, Y)=K Y-V(\varphi)
$$

Note that in this linear velocity model the pressure is nonetheless inhomogeneous via the lapse function $Y=\varphi_{, t} / N\left(t, x^{i}\right)$.

For the model (12)

$$
V=3\left[H^{2}+\frac{k}{a^{2}}\right]
$$

Combining (2), (9) and (12) implies the Hubble expansion is directly related to the potential:

$$
3 K H=-\partial V / \partial \varphi
$$

Taking the partial time derivative of (13), and using (14),

$$
\varphi_{, t}=\frac{2}{K}\left[\frac{k}{a^{2}}-H_{, t}\right]
$$

Hence, given $a(t)$ equations (13) and (15) allow one to reconstruct the potential (at least in parametric form). For the power law expansion $a(t)=\left(t / t_{0}\right)^{n}$

$$
\begin{aligned}
& V(t)=3\left[\frac{n^{2}}{t^{2}}+k\left(\frac{t_{0}}{t}\right)^{2 n}\right] \\
& \varphi(t)=-\frac{2}{K}\left[\frac{n}{t}+\frac{k t_{0}}{2 n-1}\left(\frac{t_{0}}{t}\right)^{2 n-1}\right]
\end{aligned}
$$

In the de Sitter-like case $a(t)=\mathrm{e}^{H\left(t-t_{0}\right)}$

$$
\begin{aligned}
& V(t)=3\left[H^{2}+k \mathrm{e}^{-2 H\left(t-t_{0}\right)}\right] \\
& \varphi(t)=-\frac{k}{K} \mathrm{e}^{-2 H\left(t-t_{0}\right)} \\
& \therefore V(\varphi)=3\left(H^{2}-K \varphi\right)
\end{aligned}
$$

\section{K-Essence from 5-D and Non-Linear "Electrodynamics"/Branes}

Albeit the model (12) has the requisite properties to serve as the source in the Stephani universe, one seems to have traded one mystery for another: how is one to understand the linear dependence on $Y$ ? To answer this we recall that long before Kaluza and Klein, Nordstrom [17] proposed to obtain a scalar gravity theory from 5-dimensional "electrodynamics" by applying a "cylinder condition". More specifically, let the 5-dimensional co-ordinates be denoted by $x^{M}=\left(x^{\mu}, y\right)$ and for the 5-vector potential $A_{M}=\left(A_{\mu}, \varphi\right)$; assuming $A_{M}$ is independent of the fifth co-ordinate, the 5-dimensional field strength $F_{M N}^{(5)}=A_{N, M}-A_{M, N}$ decomposes as $F_{\mu \nu}^{(5)}=F_{\mu \nu}, F_{\mu 5}^{(5)}=\varphi_{, \mu}$. Then taking the 5-dimensional metric $g_{M N}^{(5)}$ of the form $g_{\mu \nu}^{(5)}=g_{\mu \nu}, g_{\mu 5}^{(5)}=0, g_{55}^{(5)}=-1$, we have: 


$$
-\frac{1}{2} F^{(5)} \cdot F^{(5)} \equiv-\frac{1}{2} F_{M N}^{(5)} F^{(5) M N}=-\frac{1}{2} F_{\mu \nu} F^{\mu v}+g^{\mu v} \varphi_{, \mu} \varphi_{, v}=-\frac{1}{2} F \cdot F+Y^{2}
$$

As $[A \bullet A]^{(5)}=A_{M} A^{M}=A_{\mu} A^{\mu}-\varphi^{2}=A \bullet A-\varphi^{2}$, for compact $y$ it follows that any k-essence model $\mathcal{L}(\varphi, Y)$ can be obtained from a 5 -dimensional model ${ }^{3}$ $\mathcal{L}^{(5)}\left(\sqrt{-[A \bullet A]^{(5)}}, \sqrt{-\frac{1}{2} F^{(5)} \cdot F^{(5)}}\right)$ by dimensional reduction provided we also set $A_{\mu}=0$.

Taking the range of the fifth co-ordinate as $0 \leq y \leq l_{5}$, for our model source in the Stephani universe

$$
l_{5} \mathcal{L}^{(5)}=K \sqrt{-\frac{1}{2} F^{(5)} \cdot F^{(5)}}-V\left(\sqrt{-[A \bullet A]^{(5)}}\right)
$$

Similar kinetic terms appear in the context of nonlinear Born-Infeld electrodynamics and D-branes in string/M-theory. Of particular note is that Nielson and Oleson [18] proposed a Lagrangian density of the form $\sqrt{-\frac{1}{2} F \cdot F}$ as a field theory for closed dual strings identified as magnetic field lines. In our case $F_{05}^{(5)} \neq 0$ is electric but its dual is the Kalb-Ramond field strength $H_{i j k}$ that is purely magnetic [19]. We thus suggest that the kinetic part of (19) be understood as originating in string/M-theory space filling magnetic branes.

\section{Conclusion}

In this paper, we have considered the issue of the matter source in the Stephani universe as an inhomogeneous alternative to the FRW model with a cosmological constant. We have shown that a form of k-essence has the requisite properties to be that source, and that this k-essence can be obtained by dimensional reduction of a 5-dimensional model truncation of string/M-theory.

\section{Acknowledgements}

This work was supported by a grant from the National Research Foundation.

\section{Conflicts of Interest}

The authors declare no conflicts of interest.

\section{References}

[1] Perlmutter, S., et al. (1999) Measurements of $\Omega$ and $\Lambda$ from 42 High-Redshift Supernovae. The Astrophysical Journal, 517, 565.

[2] Riess, A.G., et al. (1998) Observational Evidence from Supernovae for an Accelerating Universe and a Cosmological Constant. The Astrophysical Journal, 116, 1009.

[3] Weinberg, S. (1989) The Cosmological Constant Problem. Reviews of Modern Physics, 61, 1. https://doi.org/10.1103/RevModPhys.61.1

${ }^{3}$ The appearance of $[A \cdot A]^{(5)}$ as such breaks gauge invariance, but can be understood as the unitary gauge limit of $\left[A^{(\theta)} \cdot A^{(\theta)}\right]^{(5)}, A_{M}^{(\theta)}=A_{M}+\theta_{, M}$ with $\theta$ the Stueckelberg field. 
[4] Clarkson, C., Ellis, G., Larena, J. and Umeh, O. (2011) Does the Growth of Structure Affect Our Dynamical Models of the Universe? The Averaging, Backreaction, and Fitting Problems in Cosmology. Reports on Progress in Physics, 74, Article ID: 112901. https://doi.org/10.1088/0034-4885/74/11/112901

[5] Redlich, M., Bolejko, K., Meyer, S., Lewis, G.F. and Bartelmann, M. (2014) Probing Spatial Homogeneity with LTB Models: A Detailed Discussion. Astronomy \& Astrophysics, 570, A63. https://doi.org/10.1051/0004-6361/201424553

[6] Adamek, J., Clarkson, C., Durrer, R. and Kunz, M. (2014) Does Small Scale Structure Significantly Affect Cosmological Dynamics? Physical Review Letters, 114, Article ID: 051302.

[7] Tolman, R.C. (1934) Relativity, Thermodynamics and Cosmology. Oxford University Press, Cambridge.

[8] Wyman, M. (1946) Equations of State for Radially Symmetric Distributions of Matter. Physical Review, 70, 396. https://doi.org/10.1103/PhysRev.70.396

[9] Stephani, H. (1967) Über Lösungen der Einsteinschen Feldgleichungen, die sich in einen fünfdimensionalen flachen Raum einbetten lassen. Communications in Mathematical Physics, 4, 137-142. https://doi.org/10.1007/BF01645757

[10] Sedigheh Hashemi, S., Jalalzadeh, S. and Riazi, N. (2014) Dark Side of the Universe in the Stephani Cosmology. European Physical Journal C, 74, 2995.

[11] Armendariz-Picon, C., Mukhanov, V. and Steinhardt, P.J. (2000) Dynamical Solution to the Problem of a Small Cosmological Constant and Late-Time Cosmic Acceleration. Physical Review Letters, 85, 4438. https://doi.org/10.1103/PhysRevLett.85.4438

[12] Bilic, N., Tupper, G.B. and Viollier, R.D. (2009) Cosmological Tachyon Condensation. Physical Review D, 80, Article ID: 023515.

https://doi.org/10.1103/PhysRevD.80.023515

[13] Sen Mod, A. (2002) Field Theory of Tachyon Matter. Physics Letters A, 17, 1797-1804. https://doi.org/10.1142/S0217732302008071

[14] Bilic, N., Tupper, G.B. and Viollier, R.D. (2002) Unification of Dark Matter and Dark Energy: The Inhomogeneous Chaplygin Gas. Physics Letters B, 535, 17-21. https://doi.org/10.1016/S0370-2693(02)01716-1

[15] Scherrer, R.J. (2004) Purely Kinetic k Essence as Unified Dark Matter. Physical Review Letters, 93, Article ID: 011301. https://doi.org/10.1103/PhysRevLett.93.011301

[16] De-Santiago, J., Cervantes-Cota, J.L. and Wands, D. (2013) Cosmological Phase Space Analysis of the $F(X)-V(\Phi)$ Scalar Field and Bouncing Solutions. Physical Review $D$, 87, Article ID: 023502. https://doi.org/10.1103/PhysRevD.87.023502

[17] Nordstrom, G. (1914) Über die Möglichkeit, das elektromagnetische Feld und das Gravitationsfeld zu vereinigen. Physikalische Zeitschrift, 15, 504.

[18] Nielsen, H.B. and Oleson, P. (1973) Local Field Theory of the Dual String. Nuclear Physics B, 57, 367-380. https://doi.org/10.1016/0550-3213(73)90107-7

[19] Bilić, N., Tupper, G.B. and Viollier, R.D. (2007) Chaplygin Gas Cosmology-Unification of Dark Matter and Dark Energy. Journal of Physics A, 40, 6877. https://doi.org/10.1088/1751-8113/40/25/S33 